\title{
Study of clay soils swelling by the new method based on Laser Interferometry and the classical Odometer test
}

\section{Etude du gonflement des sols argileux par la nouvelle méthode basée sur I'Interférométrie Laser et l'essai classique par l'Odomètre}

\author{
Hassan ASSELMAN ${ }^{1}$ andAouatefDAOUIDI ${ }^{1}$ \\ ${ }^{1}$ AbdelmalekEssaadiUniversity, Science Faculty, Optics and Photonics Laboratory, Tetouan, Morocco.
}

\begin{abstract}
Geotechnical engineering participates in the act of construction, which means that it must meet a double concern for securityand economy. Therefore, an essential part of the engineer's responsibility rests on the recognition of soils in order to determine their nature and properties (taking into account the flow of water). In the present work, we measure the swelling and the permeability by detection of the swelling, by our new optical method based on the interferometry-laser, invented by Hassan Asselman, within the team of Optics and photonics of sciences faculty, Tetouan- Morocco. This new prototype allows us to directly measure the following parameters: the permeability $\mathrm{k}(\mathrm{m} / \mathrm{s})$, the Young module Eand the swelling index Cs. For the latter parameter, the evolution of the strain as a function of the stresses $\sigma(\mathrm{Pa})$ is measured for a given degree of saturation (Until saturation). Moreover, we will use the classical odometer test, which reproduces the conditions of deformation of the soils. Using the results of the latter by the graphic methods of Taylor and Gasagrande, it is possible to determine the value of the coefficient of consolidation of the soil Cv. According to the Darcy theoretical modelfor a saturated medium, Cv depends on the permeability, the compressibility coefficient mv (or the inverse of the model of Young odometric) and the voluminal weight of the water $\gamma \mathrm{w}$. These tests will be carried out at the GEORET Geotechnical Laboratory in Tetouan. To perform this work, we chose a sample of claydistrubed, already characterized by X-ray diffraction (whose clay fraction is illite). It is extracted from the so-called "Teffalin" quarry of the Tetouan region, used in the manufacture of pottery. Finally we give a comparison between our new patented method and the classic Odometric test
\end{abstract}

Résumé. L'ingénierie géotechnique participe à l'acte de construction, ce qui signifie qu'elle doit répondre à un double souci de sécurité et d'économie. Par conséquent, une part essentielle de la responsabilité de l'ingénieur repose sur la reconnaissance des sols afin de déterminer leurs natures et leurs propriétés (en prenant en compte l'écoulement de l'eau). Dans le présent travail, nous mesurons le gonflement et la perméabilité par détection du gonflement, par notre nouvelle méthode optique basée sur l'interférométrie-laser, inventée par Hassan Asselman, au sein de l'équipe d'Optique et photonique à la faculté des sciences, Tétouan-Maroc. Ce nouveau prototype nous permet de mesurer directement les paramètres suivants : la perméabilité $\mathrm{k}(\mathrm{m} / \mathrm{s})$, le module d'Young E et l'indice de gonflement Cs. Concernant ce dernier paramètre, on mesure l'évolution de la déformation en fonction des contraintes $\sigma(\mathrm{Pa})$ pour un degré de saturation donné (Jusqu'à atteindre la saturation). Par ailleurs, nous comptons utiliser l'essai classique œdométrique, qui reproduit les conditions de déformation des sols. L'exploitation des résultats de ce dernier par les méthodes graphiques de Taylor et Gasagrande, permettent de déterminer la valeur du coefficient de consolidation du sol Cv. D'après le modèle théorique de Darcy pour un milieu saturé, Cv dépend de la perméabilité, du coefficient de compressibilité mv (ou l'inverse du modèle de Young odométrique) et du poids volumique de l'eau $\gamma w$. Ces essais seront effectués au Laboratoire de Géotechnique GEORET à Tétouan. Pour réaliser ce travail, nous avons choisi un échantillon d'argile remanié, déjà caractérisé par diffraction des RX (dont la fraction argileuse est l'illite). Il est extrait de la carrière dite «Téffaline», de la région de Tétouan, utilisé dans la fabrication de la poterie.Enfin nous donnons une comparaison entre notre nouvelle méthode brevetée et l'essai classique Odométrique 


\section{Introduction}

En Génie Civil, les phénomènes de retrait-gonflement de certains sols argileux et des formations géologiques argileuses induisent des tassements différentiels qui se manifestent par des désordres affectant principalement les structures construites en surface (bâtiment, fondation superficielle, ouvrage de soutènement, remblais,...) et les ouvrages enterrés (tunnels, pieux, canalisation, fondation profondes,....) [1].

La détermination des comportements de gonflement et de retrait des matériaux peu perméables, dans notre cas les sols argileux, est une étape incontournable dans la conception de nombreux ouvrages de génie civil.

Pour avoir une vision claire sur ces phénomènes plusieurs paramètres peuvent être déterminés in situ ou en laboratoire. Dans cette deuxième situation, il existe une variété de tests et de méthodes visant à évaluer les propriétés hydrauliques [1]. Dans le présent travail les essais ont été réalisés moyennant la nouvelle méthode inventée dans notre laboratoire [2, 3], par Hassan Asselman[4, 5], qui permet de mesurer : le potentiel du gonflement (ou gonflement libre), le gonflement sous une contrainte et la perméabilité des sols argileux. Ce nouveau prototype possède des qualités, des atouts et des champs d'application très larges qui le rendent très attractif, facile à mettre en œuvre et à commercialisation dans un futur proche. Le RROB (Rapport de Recherche avec Opinion sur le Brevet) élaboré par 1'OMPIC indique que le brevet d'invention $\mathrm{N}^{\circ} 1.586 .243 \mathrm{du}$ Français Bernard CORVAISIER déposé le 1er Octobre 1968 sous le titre : "Appareillage pour étudier le gonflement d'un échantillon de sol » (Odomètre), déposant : Etat Français, représenté par le ministre de l'équipement et du logement et laboratoire central des ponts et chaussées, résident en France (Paris) et qui est utilisé par les bureaux d'études du monde entier pour l'analyse des sols Argileux, s'avère efficace, mais très fastidieux et lent. Depuis cette date (1968) il n'y a pas eu d'évolution! D'où l'énorme importance de notre prototype [3,4], par son gain de temps incroyable (de quelques heures au lieu de quelques semaines) et sa précision. La conclusion du rapport de préfaisabilité $\mathrm{N}^{\circ}$ LLRA077-12 Moubtakir [6] réalisé par CERIMME (Centre d'étude et de Recherche des Industries Métallurgiques, Mécaniques, Electriques et Electroniques) et financé par l'OMPIC souligne que ce nouveau prototype est techniquement facile à mettre en œuvre et sa rentabilité va dépendre de son prix de revient et de la démarche marketing qui va accompagner sa commercialisation. [7]

Les résultats présentés, s'appuient sur des essais de gonflement à charge variable dans le cas d'un milieu non saturé. Ils ont été réalisés à l'aide d'un système optique et mécanique utilisant le bras de levier.

Rappelons que le phénomène de gonflement est une augmentation de volume du matériau, quelle qu'en soit la cause. Un déchargement mécanique, une imbibition ou une sorption, une exfoliation ou des variations chimiques qui induisent une déformation positive, peuvent être la cause d'un gonflement.

Les analyses minéralogiques montrent que certaines argiles, pour lesquelles les liaisons inter feuillets sont très faibles, ont la propriété de fixer les molécules d'eau entre deux feuillets voisins. L'eau pénètre à l'intérieur des particules et s'organise en couches mono moléculaires, il s'agit alors d'un gonflement intra particulaire ou inter foliaire [1].

Elle est à noter que le phénomène de retrait consiste en une diminution du volume du sol due à l'évaporation de l'eau présente dans ses pores. L'assèchement progressif $\mathrm{du}$ sol provoque des tensions capillaires et la formation de ménisques autour des particules du sol. Les tensions capillaires sont à l'origine de contraintes de cisaillement. Plus l'eau s'évapore, plus les tensions capillaires augmentent et plus le rayon des pores diminuent (équation Jurin-Laplace). Parallèlement, la résistance à la traction du sol augmente. Quand cette résistance atteint les contraintes engendrées par les tensions capillaires, le retrait s'arrête, le volume du sol ne diminue plus.

La teneur en eau pour laquelle le retrait cesse correspond à la limite de retrait [1].

\section{Matériel et méthode}

\subsection{Dispositif pour mesurer le gonflement de sols argileux, inventée par Hassan Asselman}

Le dispositif expérimental que nous avons utilisé est une adaptation de l'interféromètre de Michelson caractérisé par divers application [8].

Enfin l'apparition de la nouvelle et originale application de cet interféromètre, permettant d'étudier le phénomène de gonflement-retrait ainsi que la perméabilité $[3,4,7]$ d'un échantillon de sol argileux. Cette méthode a été présentée pour la première fois dans [9].

Le schéma du dispositif expérimental est représenté sur la figure 1. Il comprend [3] :

Un interféromètre de Michelson

Un laser He-Ne de longueur d'onde $\lambda=632,8 \mathrm{~nm}$ et de puissance $1 \mathrm{~mW}$

Une lentille de courte distance focale $(2 \mathrm{~cm})$ pour observer les franges d'interférences

Une éprouvette (gouttière) en verre placée verticalement au-dessus de l'échantillon pour contrôler la teneur en eau (distillée).

Un système de détection, constitué d'une caméra reliée à l'ordinateur, un logiciel permettant de suivre au cours du temps, le défilement des franges d'interférences et leur comptage N. Cela nous permet de déterminer la déformation (gonflement).

Pour réaliser ce travail, nous avons choisi un échantillon d'argile remanié, déjà caractérisé par diffraction des RX (dont la fraction argileuse est l'illite) [4]. Il est extrait de la carrière dite «Téffaline», de la région de Tétouan, utilisé dans la fabrication de la poterie. L'échantillon a la forme d'un parallélépipède, séché, de longueur $\mathrm{L}=73.00$ $\mathrm{mm}$, de largeur $\mathrm{l}=18.00 \mathrm{~mm}$, de hauteur $\mathrm{H}=6.00 \mathrm{~mm}$ et de masse $\mathrm{m}=20.10 \mathrm{~g}$. Il est façonné de manière à retenir les gouttes d'eau à sa surface pour contrôler la teneur en eau (Figure 2)[3]. La fraction de l'échantillon humidifiée de longueur $\mathrm{L}_{\mathrm{H}}=53 \mathrm{~mm}$, de largeur $\mathrm{l}_{\mathrm{H}}=3 \mathrm{~mm}$ et de hauteur $\mathrm{H}_{\mathrm{H}}=3 \mathrm{~mm}$. 
Pour éviter les vibrations mécaniques, nous avons choisi d'utiliser le bras de levier de l'interféromètre en contact direct avec l'échantillon et à travers lequel on exerce les différentes contraintes $\sigma(\mathrm{Kpa})$.

L'échantillon est placé sur un support horizontal, perpendiculairement au bras de levier et encastré à l'autre extrémité sur un support vertical fixe. La longueur du bras de levier est de $80 \mathrm{~mm}$.Le miroir mobile est situé à 8 $\mathrm{mm}$ de son axe de rotation. Le point de contact de l'échantillon avec ce bras est situé à $48 \mathrm{~mm}$. Nous avons donc une réduction de ce bras de $\mathrm{R}=1 / 6$. Par conséquent la variation de longueur effective de l'échantillon suite à sa déformation s'écrit :

$$
\Delta \mathrm{L}=\frac{\mathrm{N} \lambda}{2 \mathrm{R}}
$$

$\mathrm{N}$ est le nombre de franges d'interférences défilées de même nature.

Pour exercer des contraintes sur l'échantillon, on utilise des masses marquées de quelques centaines de grammes. Elles sont accrochées à in fil horizontal, inélastique et entourant une poulie. Le fil est attaché au bras de levier à $80 \mathrm{~mm}$ de son l'axe de rotation du bras de levier.

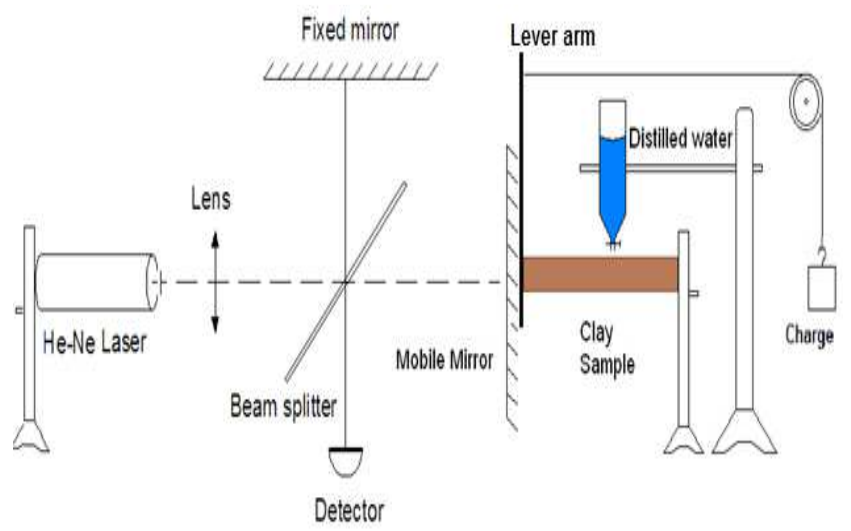

Figure 1: Schéma du dispositifexperimental

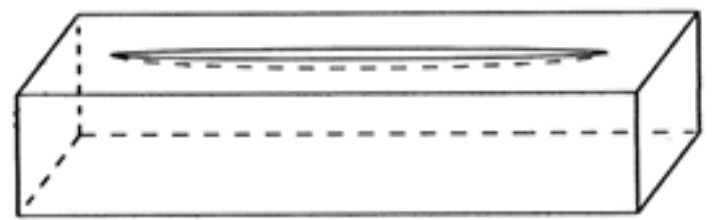

Figure 2: Schéma de l'échantillon argileux remanié

\subsection{Procédure de l'expérimentation}

La teneur en eau apparente de la fraction humidifiée de l'échantillon est estimée à $62 \%$ pour un volume d'eau $\mathrm{Ve}=1 \mathrm{ml}$. A la fin du processus du gonflement et après avoir effectué la pesée, environs $50 \%$ de cette eau s'est évaporé. La teneur en eau effective est de l'ordre de $32 \%$ [3].

Lorsqu'on injecte le volume $\mathrm{Ve}=1 \mathrm{ml}$, à la surface d'un échantillon de sol argileux, celui-ci se gonfle et le miroir se déplace. On observe le défilement des franges d'interférences et on procède au comptage de leur nombre $\mathrm{N}$ durant le temps $\mathrm{t}$ que dure le gonflement (environ quelques dizaine de minutes). On peut déterminer le gonflement sous une contrainte constante $\sigma$ (kPa)..

La contrainte totale (ressort du bras de levier et les masse marquées) appliquée sur la surface de l'échantillon est donnée par :

$$
\sigma=\frac{\mathrm{mg}}{\mathrm{S}} \frac{5}{3}+\sigma_{\mathrm{i}}
$$

$\sigma \mathrm{i}=(4.63 \pm 0.02) \mathrm{kPa}$, est la contrainte exercée par le ressort du bras de levier. Elle maintient en permanence le contact entre le bras et l'échantillon, ce qui permet la détection du gonflement. D'où l'importance de l'utilisation de ce bras.

Nous avons choisi de travailler avec les contraintes $(12,35 \pm 0.04) \mathrm{kPa},(20.06 \pm 0.07) \mathrm{kPa}$ et une teneur en eau fixe. Pour chaque valeur deo nous étudions la cinétique, c'est-à-dire l'évolution du gonflement sous pression au cours du logarithme du temps en seconde [11].

La surface $\mathrm{S}$ de l'échantillon subissant la contrainte horizontale est de $108 \mathrm{~mm} 2$. Le choix d'une petite surface est dans le but d'atteindre les pressions exercées dans le cas d'un essai odométrique (environ100 KPa) pour une éventuelle comparaison avec cette méthode classique.

Le gonflement sous pression (\%) est donnée par :

$$
\frac{\Delta \mathrm{L}}{\mathrm{L}_{\mathrm{H}}}=\frac{\mathrm{N} \lambda}{2 \mathrm{~L}_{\mathrm{H}} \mathrm{R}}(\%)
$$

L'indice de gonflement est donné par la relation suivante [11]:

$$
\mathrm{C}_{\mathrm{S}}=\frac{\Delta \mathrm{e}}{\Delta \log (\sigma)} \quad \%
$$

$\Delta \mathrm{e}$ : est la variation de l'indice des vides de l'échantillon

L’indice des vides e s'écrit :

$$
e=e_{i}+\frac{\Delta L}{L_{S}}
$$

$\mathrm{e}_{\mathrm{i}}=0,66$ (indice des vide initial), mesuré par le pycnomètre et l'imprégnation à sec.

$\Delta \mathrm{L}=\mathrm{L}_{0}-\mathrm{L}$ (déformation latérale ou gonflement)

$\mathrm{L}_{0}$ est la longueur initiale de l'échantillon séché $\mathrm{L}_{\mathrm{s}}=0,032 \mathrm{~m}$ (la longueur du solide)

On utilise le poids volumique du solide $\gamma_{\mathrm{s}}=26,5 \mathrm{KN} / \mathrm{m}^{3}$

\section{Résultats et discussions}

\subsection{Gonflement-retrait sous la contrainte $\sigma(\mathrm{kPa})$}

\subsubsection{Teneur en eau fixée $(\mathrm{Ve}=1 \mathrm{ml})$}


La figure 3, représente nos résultats expérimentaux, concernant l'évolution du gonflement et retrait au cours $\mathrm{du}$ temps, pour les contraintes $12,35 \mathrm{kPa}$ et $20,35 \mathrm{kPa}$ avec $\mathrm{Ve}=1 \mathrm{ml}, \mathrm{T}=20^{\circ} \mathrm{C}$, Humidité $\mathrm{h}=90 \%$

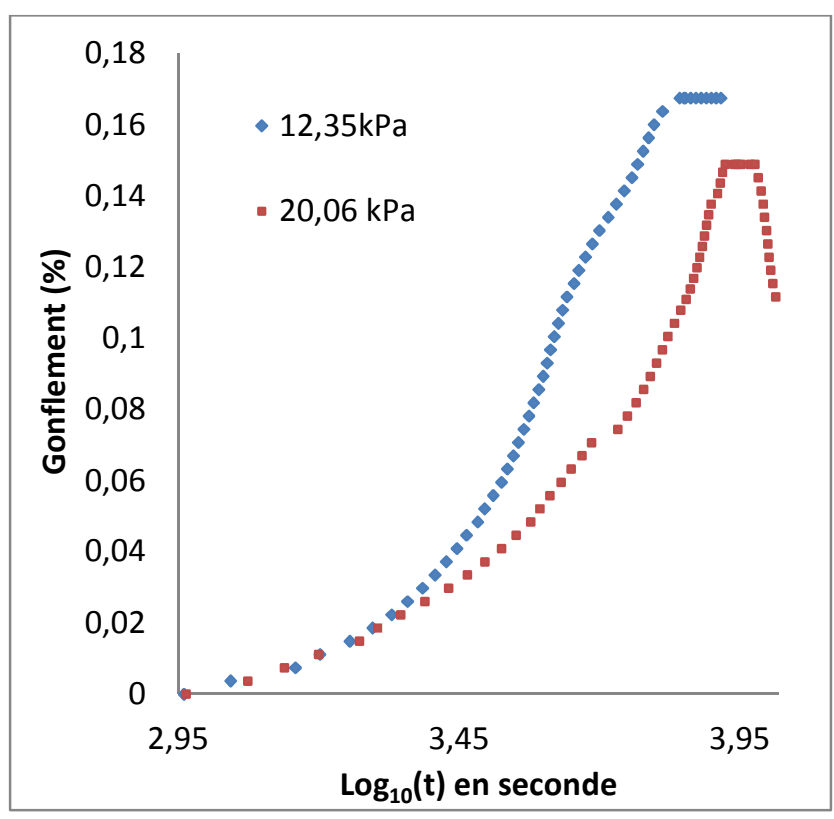

Figure 3: Cinétique du gonflement (\%) en fonction $\log _{10}(t)(s)$, pour $\sigma=12,35$ et $\sigma=20,06 \mathrm{kPa}$

De nombreux travaux expérimentaux font apparaître ce type de cinétique. Il se traduit par un gonflement primaire et un gonflement secondaire [10].

L'allure de la courbe obtenue relative à $\sigma=20,06 \mathrm{kPa}$ est en bon accord avec celle rencontrée dans la littérature $[10,12]$. Elle atteint la stabilisation au bout de $140 \mathrm{~min}$ environ. La partie constante de la courbe où le défilement des franges s'arrête, dure environ $18 \mathrm{~min}$. Audelà des $158 \mathrm{~min}$ le phénomène de retrait commence. On observe alors le défilement des franges d'interférences dans le sens contraire.

Sachant que le processus de retrait est très lent. Il dépend des conditions du milieu ambiant (température et humidité), nous avons été amenés à chauffer notre laboratoire à une température voisine de $32^{\circ} \mathrm{C}$ pour accélérer le processus du retrait et le mettre en évidence. Le retrait complet jusqu'à stabilisation peut être observé à une température ambiante mais cela nécessiterait de laisser le laser allumé pendant quelques heures. Cela permettrait de voir si le processus est réversible.

En ce qui concerne l'effet de la contrainte (KPa) sur le sol argileux humidifie en phase de gonflement. En analysant les deux courbes, nous relevons les observations suivantes :

- Nous constatons une superposition des deux courbes durant la première phase (environ 62min). Le gonflement observé est donc indépendant de la contrainte $\sigma$ exercée.

- Durant la deuxième phase un décalage temporel $\Delta t$ (sec) est observé pour chaque valeur de N. Ceci est dû à l'influence de la contrainte $\sigma$. En effet la contrainte appliquée provoquerait le ralentissement du processus de pénétration des molécules d'eau entre les feuillets d'argile. La valeur maximale de cet écart $\Delta \mathrm{t}$ est atteinte pour $\mathrm{N}=32$ (Figure 4).

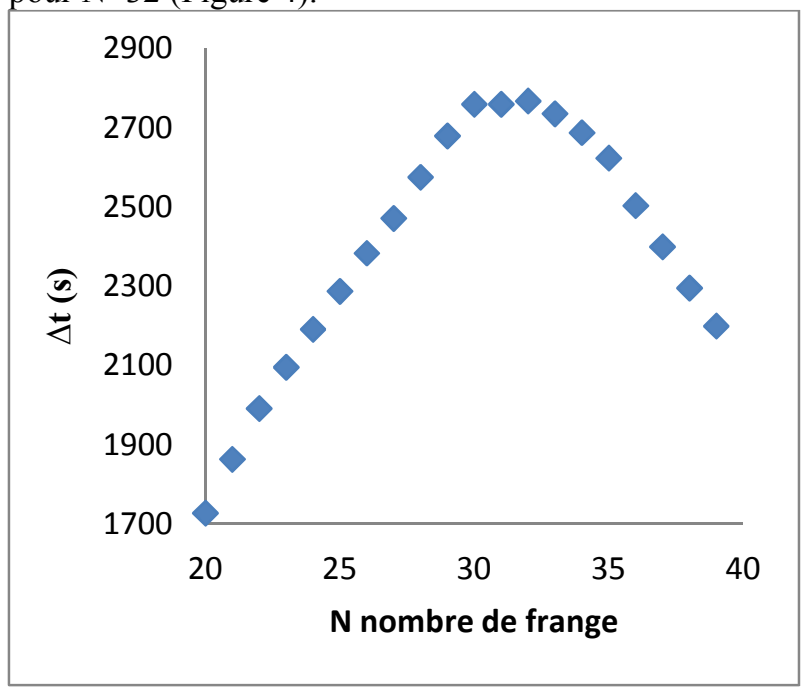

Figure 4 : L'écart temporel $\Delta t$ (sec) entre les deux courbes en fonction de $\mathrm{N}$

- Lorsque la contrainte augmente, le nombre total des franges défilées diminue sensiblement (tableau 1). Ce résultat est en bon accord avec la littérature [11].

Le tableau 1 résume nos résultats obtenus.

Tableau 1. Gonflement et sa duration en fonction des contraintes oappliquées

\begin{tabular}{|c|c|c|c|}
\hline$\sigma(\mathrm{kPa})$ & $\begin{array}{c}\text { Gonflement } \\
(\%)\end{array}$ & $\begin{array}{l}\text { Indice des } \\
\text { vides : e }\end{array}$ & $\begin{array}{c}\text { Durée du } \\
\text { gonflement } \\
\text { (minutes) }\end{array}$ \\
\hline $4,63(0 \mathrm{Gr})$ & - & 0,6632681 & \\
\hline $12,35(50 \mathrm{Gr})$ & $0,167 \pm 0,004$ & 0,66266963 & 115. \\
\hline $20.06(100 \mathrm{Gr})$ & $0,149 \pm 0,004$ & 0,662373 & 140 \\
\hline
\end{tabular}

La figure 5, représente la variation de l'indice des vides en fonction du Logarithme décimal des contraintes $\sigma$. Nous obtenons la valeur de l'indice de Gonflement $\mathrm{C}_{\mathrm{s}}=$ 0,0014 (au voisinage de la saturation), en un temps record d'environs 4 heures (au lieu de quelques semaines par la méthode classique Odométrique). Ce résultat est significatif puisque l'Illite est une argile non gonflante (la

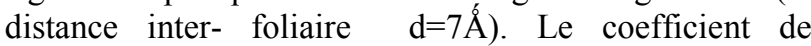
corrélation de la droite $\mathrm{R}^{2}=1$. 


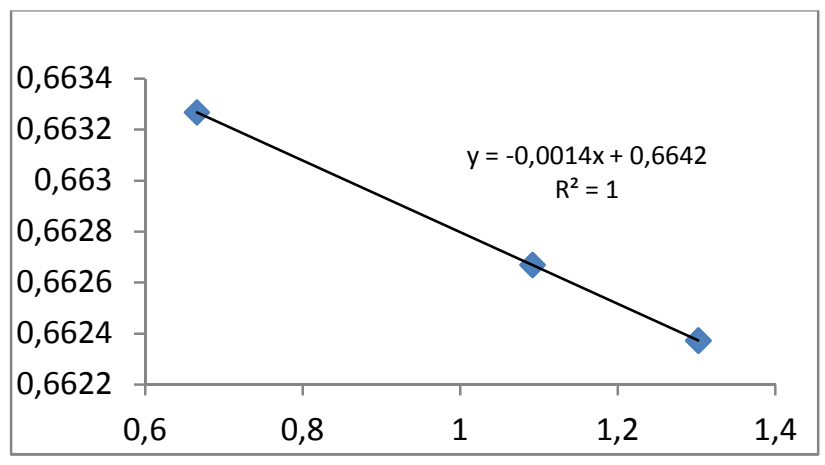

Figure 5 : Indice des vides en fonction de Log10 des Contraintes ( $\mathrm{kPa})$

\subsubsection{Teneur en eau fixée $(V e=0,5 \mathrm{ml})$}

La figure 6, représente nos résultats expérimentaux, concernant l'évolution du gonflement au cours du logarithme décimal du temps en secondes, pour les contraintes $53,29,58,2$ et $66,61 \mathrm{kPa}$ avec $\mathrm{Ve}=0,5 \mathrm{ml}$, $\mathrm{T}=20^{\circ} \mathrm{C}$, Humidité $\mathrm{h}=90 \%$

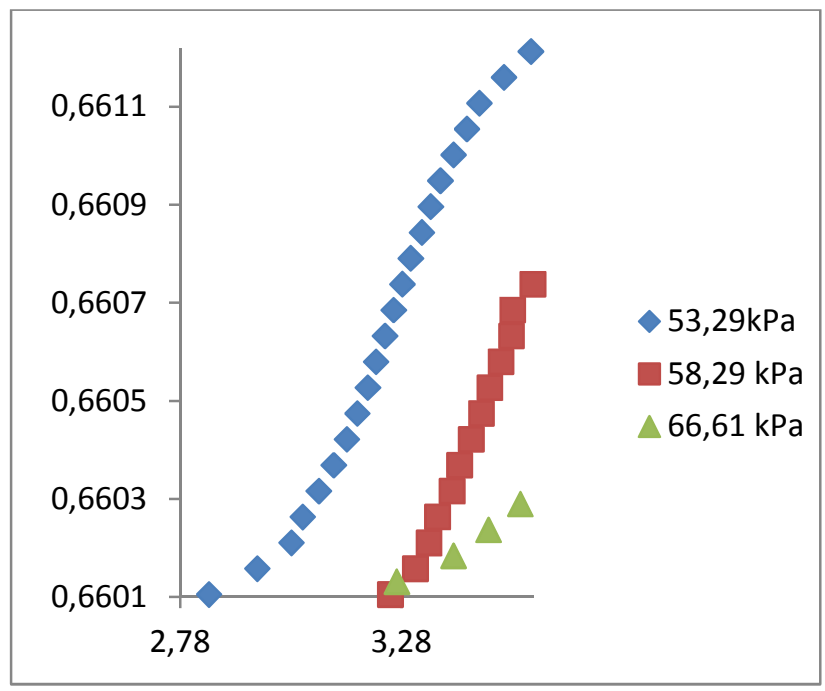

Figure 7 : Indice des vides en fonction du Logarithme décimal du temps $(\mathrm{Ve}=0,5 \mathrm{ml})$

La figure 7, représente la variation de l'indice des vides en fonction du Logarithme décimal des contraintes $\sigma$.

Nous obtenons la valeur de l'indice de Gonflement $\mathrm{C}_{\mathrm{s}}=$ 0,015 . Le coefficient de la droite est de 0,97 . On constate que lorsque la teneur en eau diminue l'indice de gonflement $\mathrm{C}_{\mathrm{s}}$ augmente de façon significative ;

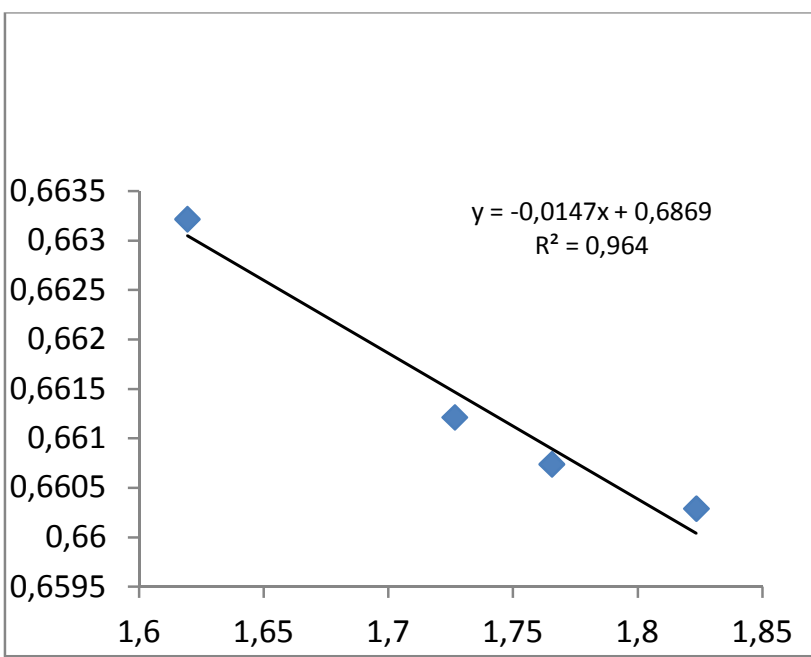

Figure 6 : Indice des vides en fonction de Log10 des Contraintes ( $\mathrm{kPa})$

\subsection{Comparaison entre notre nouvelle méthode et la méthode classique}

Pour pouvoir faire cette étude comparative importante, il nous a été nécessaire de maitriser la méthode classique sur tous les aspects : procédé de mesure et exploitation des résultats (graphique, signification des paramètres ....)

\subsubsection{Exemple d'un essai classique odométrtrique (milieu saturé.}

Le bureau d'études géotechniques (GEORET), nous a fourni les résultats d'un essai œdométrique d'une montmorillonite, prélevée à l'ENSA de Tétouan. On obtient les résultats suivants:

$\mathrm{C}_{\mathrm{c}}=0,13$ (Selon la norme on a un sol moyennant compressible)

$\mathrm{C}_{\mathrm{S}}=0,04 \quad$ (Présence d'une tendance au gonflement)

La valeur de la perméabilité $\mathrm{K}=2,510^{-11} \mathrm{~m} / \mathrm{s}$ (milieu saturé) est très faible.

D'après le modèle théorique de Darcy, la Perméabilité d'un milieu saturé s'écrit:

$$
k(m / s)=C_{v} m_{v} \gamma_{w}
$$

$\mathrm{m}_{\mathrm{v}}$ : Coefficient de compressibilité (ou l'inverse du modèle de Young œdométrique)

$\mathrm{C}_{\mathrm{v}}$ : Coefficient de Consolidation $\gamma_{\mathrm{w}}$ :Poids volumique de l'eau.

Le résultat de l'essai classique a été obtenu au bout de trente jours!

\subsubsection{Perméabilité d'une montmorillonite par la nouvelle méthode (milieu non saturé)}

Récemment nous avons mesuré la perméabilité d'une montmorillonite [7]. On a obtenu le résultat suivant: $\mathrm{K}=3,3310^{-4} \mathrm{~m} / \mathrm{s}$.

Ce résultat a été obtenu au bout d'une heure. Nous avons travaillé avec un échantillon remanié, séché à l'étuve à $105^{\circ} \mathrm{C}$ pendant 24 heures. 
Dans un milieu non saturé, la Perméabilité est donnée par l'équation Brooks and Corey [13]:

$$
K=K_{s}\left(\frac{\theta-\theta_{0}}{\theta_{s}-\theta_{0}}\right)^{\alpha}
$$

Nous mis en évidence par notre méthode expérimentale, que la perméabilité augmente avec l'augmentation de la teneur en eau. Ceci est en bon accord avec l'équation (7). Lorsqu'on s'approche de la saturation, il se produit une chute brutale de la valeur de $\mathrm{K}$ d'un facteur d'environs $10^{-7}$

\subsubsection{Tableau comparatif entre les deux méthodes expérimentales}

La comparaison entre les deux méthodes est indiquée sur le tableau 2

Tableeau 2. Comparaison entre les deux méthodes.

\begin{tabular}{|c|c|c|}
\hline $\begin{array}{c}\text { Méthode de } \\
\text { mesure }\end{array}$ & Odomètre & $\begin{array}{c}\text { Interférométrie } \\
\text { laser }\end{array}$ \\
\hline Milieu & Saturé & $\begin{array}{c}\text { Non saturé ou } \\
\text { presque saturé }\end{array}$ \\
\hline $\begin{array}{c}\text { Lieu de } \\
\text { mesure }\end{array}$ & Laboratoire & $\begin{array}{c}\text { Laboratoireou } \\
\text { In-situ }\end{array}$ \\
\hline $\begin{array}{c}\text { Indice de } \\
\text { compression } \\
\text { Cc }\end{array}$ & Oui & Non \\
\hline $\begin{array}{c}\text { Indice de } \\
\text { gonflement Cs }\end{array}$ & Oui & Oui \\
\hline $\begin{array}{c}\text { Perméabilité } \\
\text { K(m/s) }\end{array}$ & Milieu saturé & $\begin{array}{c}\text { Non saturé ou } \\
\text { presque saturé }\end{array}$ \\
\hline $\begin{array}{c}\text { Durée de } \\
\text { mesure }\end{array}$ & Quelquessemaines & $\begin{array}{c}\text { Maximum } \\
\text { quatreheures }\end{array}$ \\
\hline Précision & - & $3 \%$ \\
\hline
\end{tabular}

On voit clairement que notre méthode possède des avantages surtout le record en temps d'obtention des mesures ainsi que la précision ;

\section{Conclusion}

Ce travail nous a permis de mesurer le gonflement-retrait, d'un sol argileux de façon rapide et simple, grâce à l'utilisation de notre nouvelle méthode basé sur l'interférométrie laser. Nous avons réussi à coupler les deux systèmes optique et mécanique, d'où l'originalité de notre travail. On a pu mettre en évidence artificiellement le phénomène de retrait. De plus nous avons observé l'effet de la contrainte $\sigma(\mathrm{kPa})$ sur un échantillon d'argile humidifié (teneur en eau fixée), s'opposant au phénomène du gonflement de l'échantillon. En effet lorsque la pression sur l'échantillon augmente le nombre de franges défilées diminue et sa durée augmente sensiblement. Ce résultat nous a permis la détermination de l'indice de gonflement $\mathrm{C}_{\mathrm{s}}$ dans le cas de l'Illite en un temps record. Ce dernier paramètre dépend de la teneur en eau. D'où l'originalité de notre travail.
Enfin nous donnons le tableau comparatif entre les deux méthodes expérimentales.

Comme perspectives nous souhaitons normaliser notre méthode brevetée en mesurant l'indice de gonflement pour quelques échantillons déjà étudiés par la méthode classique et faire la comparaison. Pour mieux comprendre le phénomène étudié, gonflement-retrait des sols argileux et vu la complexité de ce milieu poreux (phases: liquide, solide et gazeux), nous comptons entamer une étude théorique, adaptée à notre original méthode expérimentale, en se basant sur la méthode des éléments finis (MEF), qui sera validée par nos mesures.

\section{References}

1. H. Nowamooz, Sciences de l'ingénieur [physics], Institut National Polytechnique de Lorraine - INPL, Français, (2007).

2. H. Asselman, Device for measuring the infiltration velocity the water of deep clay soil, Patent $\mathrm{N}^{\circ} \mathbf{3 5 5 5 6}$, in OPMIC (Moroccan Office of Industrial and Commercial Property), Casablanca-Morocco, (2013).

3. H. Asselman, New optical method to measure the swelling of clay soils by laser interferometry, Patent $\mathrm{N}^{\circ}$ 35229, in OPMIC (Moroccan Office of Industrial and Commercial Property), Casablanca-Morocco, (2012).

4. H. Asselman, A. Daouidi, K. Draoui, et A. Asselman, MATEC Web of Conferences, Vol. 11, (2014).

5. H. Asselman, A. Bakkali, M. Ajdour, and M. Essaaaidi, MATEC Web of Conferences 2, (2012).

6. CERIMME (Center of Study and Research of Metallurgical Industries, Mechanical, Electrical and Electronic), Pre-feasibility study, Report NLLRA077-12 Moubtakir, Casablanca-Morocco, (2012).

7. A. Daouidi, H. Asselman, J. Civil Eng. Architect. Res, Vol. 3 No. 1, (2016).

8. H. Asselman, M. El Addad, Congers COMPLES'2K7. ENSA Agadir-Morocco (2007).

9. H. Asselman, The Second day clays and Sustainable Development, Faculty of Science, Tetouan-Morocco, (2010).

10. A. Medjnoun, Doctoral Thesis, Mouloud MammeriTiziOuzouUniversity, (2014).

11. J.-P.Magnan, C. Mieussens, B. Soyez, J. Vautrain. Essais oedométriques. Ifstar, (1985).

12. Vizier, J.F., Cah. O.R.S.T.O.M., sér. Pédol., vol. IX, $\mathrm{n}^{\circ} 2,(1971)$.

13. A. MUSY, Simulation des écoulements en milieu poreux non saturé, Journal of hydrology, Elsevier scientificpublishingcompany29, pp 1-21, (1976) 